\title{
Alterações das funções de mastigação e deglutição no processo de alimentação de idosos institucionalizados
}

\author{
Changes of mastication and swallowing in the process of feeding institutionalized elderly
}

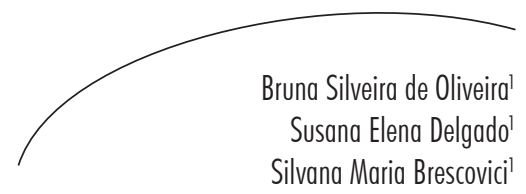

Resumo

Objetivo: Analisar o processo de alimentação de idosos institucionalizados e, mais especificamente, descrever o perfil sociodemográfico dos idosos, verificar as possíveis alterações estruturais do sistema estomatognático, das funções de mastigação e deglutição e constatar as dificuldades alimentares autorreferidas. Método: Estudo do tipo quantitativo, descritivo, observacional, transversal e prospectivo. A amostra foi constituída por 27 indivíduos do gênero feminino e três do gênero masculino, com idades entre 65 e 93 anos, residentes em uma instituição de longa permanência para idosos de Canoas-RS, no período de julho a agosto de 2012. Utilizando-se de dois protocolos, foi realizada uma entrevista, uma avaliação das estruturas do sistema estomatognático e a observação de uma refeição em ambiente habitual de alimentação do idoso. Resultados: Quanto aos aspectos alimentares, a dificuldade de mastigar algum tipo de alimento e a preferência por alimentos macios foram frequentemente citadas. As principais modificações nas estruturas estomatognáticas e em suas funções foram as alterações de mobilidade e tonicidade e a adaptação da mastigação e da deglutição, por meio da participação exagerada da musculatura perioral e do ritmo mastigatório lentificado. Conclusão: O processo de alimentação dos idosos institucionalizados sofre modificações ao longo dos anos, e a habituação das dificuldades encontradas, por meio da realização de compensações, é frequente entre eles. Os resultados evidenciam a necessidade da atuação fonoaudiológica interdisciplinar nas instituições de longa permanência para idosos, a fim de minimizar o impacto dos efeitos do envelhecimento nas funções estomatognáticas, proporcionando qualidade de vida ao idoso institucionalizado.

\section{Abstract}

Objective: Analyze the process of feeding institutionalized elderly, and, more specifically, to describe the socio-demographic profile of the elderly, to check for possible structural alterations of the stomatognathic system, the functions of chewing and swallowing and to verify self-reported feeding difficulties. Methods: Study of quantitative, descriptive, observational, transversal and prospective type. The sample consisted of 27 female and 3 male subjects, aged between 65 and 93 years, residents of a long-stay institution in

Curso de Fonoaudiologia. Universidade Luterana do Brasil. Canoas, RS, Brasil.

Palavras-chave: Idosos. Alimentação. Fonoaudiologia. 
Canoas-RS in the period between July and August in 2012. Using two protocols, we conducted an interview, an assessment of the stomatognathic system and, finally, the observation of an elderly's meal in his habitual feeding environment. Results: About the feeding difficulties, the subjects cited their difficulty in chewing any food and their preference for soft foods. The main changes in the stomatognathic structures and their functions have been changes in tone and mobility and adaptation of chewing and swallowing, through the exaggerated participation of the perioral muscles and the slowed masticatory rhythm. Conclusion: The process of feeding the institutionalized elderly undergoes changes over the years, and habituation of the difficulties encountered by performing compensation is common among them. The results highlight the need for interdisciplinary speech therapy in long-stay institutions in order to minimize the impact of the effects of aging on stomatognathic functions, providing a better quality of life to the institutionalized elderly.
Key words: Elderly. Feeding. Phonoaudiology.

\section{INTRODUÇÃOO}

O envelhecimento populacional é acompanhado por mudanças nas estruturas e nos papéis da família, comprometendo sua função de cuidar, proteger e acolher o idoso. ${ }^{1}$ Frente a esse quadro, vários são os fatores que acabam por levar o idoso à institucionalização, como os problemas familiares, de saúde, limitação das atividades diárias, situação mental, ausência de suportes sociais e pobreza. As instituições de longa permanência para idosos são estabelecimentos que têm como foco o atendimento, acolhimento e cuidado, em regime residencial, de pessoas com idade igual ou maior que 60 anos. ${ }^{2,3}$

Segundo Ribeiro, ${ }^{4}$ o processo de envelhecimento pode ser definido como natural, progressivo, degenerativo, universal e intrínseco, caracterizado por menor eficiência funcional, com enfraquecimento dos mecanismos de defesa frente às variações ambientais e perda das reservas funcionais. Sendo assim, nesse processo ocorrem alterações que podem afetar o sistema estomatognático, ocasionando distúrbios na alimentação.

Sabe-se que o envelhecimento leva a modificações na anatomia e fisiologia que, por si só, já implicam alterações na funcionalidade do sistema estomatognático. ${ }^{5}$ Quanto aos idosos institucionalizados, fatores de ordem emocional e psicológica como a solidão, devido ao isolamento social e à ausência de convívio familiar, podem acelerar essas alterações. ${ }^{6}$
A literatura cita como principais modificações: a retração da gengiva com consequentes problemas na adaptação de próteses dentárias, a diminuição do número de papilas gustativas, o decréscimo da produção salivar e a diminuição de tônus e força da língua e da musculatura mastigatória..$^{7-9}$ Quanto aos aspectos dentários, estudos têm apontado um quadro de saúde bucal precário na população idosa institucionalizada, destacando-se a alta prevalência de doenças periodontais, o número reduzido de dentes ou edentulismo, próteses mal adaptadas ou com péssima conservação e higiene bucal deficitária., ${ }^{7,8}$

Diante de todos os fatores citados, as funções estomatognáticas ficam mais lentas, descoordenadas, e adaptadas às perdas estruturais sofridas ao longo dos anos. ${ }^{10}$ Os reflexos do processo de envelhecimento na mastigação são observados na perda da capacidade de controlar o bolo alimentar e da coordenação motora na lateralização dele durante o ato mastigatório, na redução da força mastigatória e na maceração dos alimentos, processo no qual o desdentado total realiza amassamento, preparando o bolo diante das condições anatômicas que possui. ${ }^{11}$

Cardos \& Buges $^{12}$ apontam as inter-relações da saúde bucal com a mastigação, uma vez que a ausência de elementos dentários interfere na boa coesão do bolo. Com isso, o idoso passa a realizar estratégias de adaptação, como, por exemplo, a substituição na dieta de alimentos mais duros e fibrosos por alimentos menos consistentes. ${ }^{13}$ Como consequência dessas características da 
mastigação, tem-se uma deglutição ocorrendo também com adaptações, visto que essa função está relacionada a uma harmonia de fatores, inclusive, a adequada mastigação. ${ }^{12}$

Cabe ressaltar que idosos saudáveis adaptamse às mudanças anatômicas e fisiológicas próprias do envelhecimento, e que esse processo por si só não causa disfunções patológicas como a disfagia (alteração da deglutição em qualquer uma de suas fases), mas deixa o organismo mais propenso à fragilização. ${ }^{5} \mathrm{O}$ termo "idoso saudável" remete àquele que possui capacidade funcional, independentemente da presença de doenças crônicas. ${ }^{14}$

Denomina-se "presbifagia" a modificação no ato de deglutir do idoso saudável, caracterizada pelas adaptações na condução do bolo alimentar que ocorrem na fase do envelhecimento. ${ }^{14}$ Dias \& Cardoso $^{15}$ referem que essas modificações levam o idoso a se habituar às dificuldades que ocorrem no processo de deglutição, não caracterizando propriamente a presença de disfagia, mas uma menor eficiência funcional.

Este estudo justifica-se uma vez que se observa que com o crescente aumento da população idosa, surge grande demanda de pacientes institucionalizados para a área fonoaudiológica. Frente às inúmeras questões levantadas, torna-se evidente a importância de conhecer as necessidades desta população, com o intuito de fortalecer a inserção do profissional fonoaudiólogo nesse contexto, para que possa colaborar junto à equipe multidisciplinar, proporcionando a esta população qualidade de vida e uma alimentação segura e efetiva.

É de extrema importância para os profissionais que desenvolvem seus trabalhos com idosos conhecer os aspectos referentes às estruturas fisiológicas que impactam nos hábitos alimentares e, consequentemente, podendo favorecer a ocorrência de desnutrição. Portanto, o objetivo deste estudo foi analisar o processo de alimentação de idosos institucionalizados e, especificamente, descrever o perfil sociodemográfico dos idosos; verificar as possíveis alterações estruturais do sistema estomatognático; das funções de mastigação e deglutição e constatar as dificuldades alimentares autorreferidas pelos idosos.

\section{MÉTODO}

Estudo do tipo quantitativo, descritivo, observacional, transversal, prospectivo e de grupo, realizado em instituição de longa permanência para idosos, no município de Canoas-RS, no período de julho a agosto de 2012. A instituição é de caráter privado e funciona em regime residencial. Foi estudada uma amostra de conveniência, composta por 30 residentes, sendo 27 do gênero feminino e três do gênero masculino, com idades entre 65 e 93 anos.

Os critérios de inclusão foram: idade igual ou superior a 60 anos, idosos de ambos os sexos, que estivessem em regime de internação, com capacidade de comunicação compreensiva e expressiva (avaliada na aplicação da entrevista) e com independência alimentar (alimentar-se sem auxílio de outro).

Os critérios de exclusão foram: presença de alteração dos níveis de consciência, conforme registrado no prontuário do paciente; idosos que não se alimentassem por via oral; com dificuldade de compreensão das questões da entrevista; traqueostomizados, com sonda para alimentação complementar, ou com histórico de alterações neurológicas, tais como sequelas de acidente vascular encefálico, doença de Parkinson, doença de Alzheimer e sequelas de trauma cranioencefálico.

A coleta foi realizada nas dependências da instituição. Realizou-se primeiramente, de forma individual, uma entrevista e, em seguida, uma avaliação do sistema estomatognático, com base em dois protocolos utilizados por Lima et al. ${ }^{13} \mathrm{O}$ protocolo de entrevista contém questões referentes aos dados sociodemográficos gerais, dados sobre saúde e tipo de alimentação atual e dificuldades alimentares autorreferidas. $\mathrm{O}$ protocolo de avaliação aborda aspectos relacionados a morfologia, postura, tonicidade e mobilidade das estruturas estomatognáticas, aspectos dentários 
e desempenho das funções de mastigação e deglutição. Os dois últimos aspectos foram avaliados durante observação de uma refeição no ambiente habitual de alimentação dos idosos.

Os voluntários da pesquisa receberam, como benefício, orientações sobre as dificuldades que foram identificadas no momento da coleta de dados. Foi realizada ainda, junto aos idosos, uma palestra com o objetivo de orientá-los quanto à correta realização da higiene oral e sua relação com a mastigação e deglutição.

Os dados coletados foram armazenados em um banco de dados, utilizando-se o software Microsoft Excel Starter 2010. Foi realizada análise estatística descritiva (média e desvio-padrão) e aplicado o teste exato de Fisher para análise da associação significativa entre as variáveis estudadas, sendo considerados significativos valores de $\mathrm{p}<0,05$.

Antes de sua realização, a pesquisa foi aprovada pelo Comitê de Ética em Pesquisa da Universidade Luterana do Brasil, sob CAAE $\mathrm{n}^{\mathrm{o}}$ 04526412.5.0000.5349, conforme preceitua a Resolução no 196/96, do Ministério da Saúde.
Todos os participantes assinaram o Termo de Consentimento Livre e Esclarecido.

\section{RESULTADOS}

Participaram do estudo 30 idosos, na faixa etária de 65 a 93 anos, com média de idade de 82,4 anos, de ambos os gêneros, sendo 27 (90\%) do gênero feminino e três $(10 \%)$ do masculino. Eles apresentaram baixo nível de escolaridade, sendo que 19 (63,3\%) possuíam o ensino fundamental incompleto, quatro $(13,3 \%)$ não frequentaram a escola, três $(10 \%)$ apresentavam ensino médio completo, dois $(6,7 \%)$ possuíam o ensino fundamental completo, um $(3,3 \%)$ apresentou ensino médio incompleto e um $(3,3 \%)$ possuía ensino superior incompleto. Quanto à naturalidade, houve predominância para a capital do Rio Grande do Sul, Porto Alegre (23,3\%), e o restante da amostra foi proveniente de várias cidades do estado.

A tabela 1 apresenta a distribuição dos idosos quanto às características de saúde atual e fatores associados. Verificou-se que, dos indivíduos participantes a hipertensão foi a patologia mais citada.

Tabela 1. Dados sobre saúde atual e fatores associados. Canoas-RS, 2012.

\begin{tabular}{lcc}
\hline \multicolumn{1}{c}{ Saúde atual } & $\mathrm{n}$ & $\%$ \\
\hline Doenças & 21 & 70,0 \\
$\quad$ Hipertensão & 14 & 46,7 \\
Cardiopatia & 12 & 40,0 \\
Refluxo & 6 & 20,0 \\
Diabetes & 4 & 13,3 \\
Outras & 3 & 10,0 \\
Doença pulmonar & & \\
Fatores associados & & 56,7 \\
Não fez uso de nenhum & 17 & 30,0 \\
Álcool & 9 & 30,0 \\
Cigarro & 9 & \\
\hline
\end{tabular}

Resposta múltipla em todas as questões. 
Observou-se que todos os idosos pesquisados afirmaram ingerir alimentos de todas as consistências. Quanto à quantidade de refeições realizadas durante o dia, $10(33,3 \%)$ realizavam três refeições, 10 (33,3\%) realizavam quatro refeições, nove $(30 \%)$ realizavam cinco refeições e apenas um $(3,3 \%)$ realizava duas refeições por dia. Sobre as visitas familiares, 19 sujeitos $(63,3 \%)$ relataram receber visitas frequentemente, oito $(26,7 \%)$ foram visitados eventualmente e apenas três $(10 \%)$ nunca receberam visitas.

A tabela 2 apresenta os dados referentes à entrevista realizada com os idosos participantes. Ressaltam-se nos achados a dificuldade em mastigar algum tipo de alimento e a preferência por alimentos macios.

Tabela 2. Dificuldades alimentares autorreferidas pelos idosos. Canoas-RS, 2012.

\begin{tabular}{|c|c|c|c|c|c|c|}
\hline \multirow[b]{2}{*}{ Questão } & \multicolumn{2}{|c|}{ Sim } & \multicolumn{2}{|c|}{ Não } & \multicolumn{2}{|c|}{ Às vezes } \\
\hline & $\mathrm{n}$ & $\%$ & $\mathrm{n}$ & $\%$ & $\mathrm{n}$ & $\%$ \\
\hline Necessidade de temperar mais a comida & 14 & 46,7 & 13 & 43,3 & 3 & 10,0 \\
\hline Dificuldade em mastigar algum alimento & 17 & 56,7 & 8 & 26,7 & 5 & 16,7 \\
\hline Dor durante a mastigação & 1 & 3,3 & 26 & 86,7 & 3 & 10,0 \\
\hline Cansaço para mastigar & 8 & 26,7 & 22 & 73,3 & & \\
\hline Preferência por alimentos macios & 19 & 63,3 & 11 & 36,7 & & \\
\hline Engasgo durante/após a deglutição & & & 21 & 70,0 & 9 & 30,0 \\
\hline Tosse durante/ após a deglutição & & & 23 & 76,7 & 7 & 23,3 \\
\hline Cansaço ao se alimentar & 2 & 6,7 & 24 & 80,0 & 4 & 13,3 \\
\hline Sensação de comida parada na garganta & & & 27 & 90,0 & 3 & 10,0 \\
\hline $\begin{array}{l}\text { Ardor e/ou dor na garganta durante ou após } \\
\text { as refeições }\end{array}$ & & & 30 & 100,0 & & \\
\hline $\begin{array}{l}\text { Necessidade de auxiliar a descida do } \\
\text { alimento com líquido }\end{array}$ & 8 & 26,7 & 20 & 66,7 & 2 & 6,7 \\
\hline Sensação de estar cheio após as refeições & 9 & 30,0 & 14 & 46,7 & 7 & 23,3 \\
\hline
\end{tabular}

A tabela 3 apresenta as alterações mais frequentes: de postura, de morfologia, de tonicidade e mobilidade dos lábios, da língua e das bochechas. Destacam-se as alterações de mobilidade e a hipofunção das estruturas, entre os idosos pesquisados. 
Tabela 3. Alterações na morfologia, postura, tonicidade e mobilidade das estruturas de lábios, língua e bochechas. Canoas-RS, 2012.

\begin{tabular}{|c|c|c|c|}
\hline Estruturas & Avaliação & $\mathrm{n}$ & $\%$ \\
\hline \multicolumn{4}{|l|}{ Lábios } \\
\hline \multirow[t]{2}{*}{ Morfologia dos lábios } & Normal & 29 & 96,7 \\
\hline & Inferior evertido & 1 & 3,3 \\
\hline \multirow[t]{3}{*}{ Espessura dos lábios } & Normal & 11 & 36,7 \\
\hline & Superior fino & 8 & 26,7 \\
\hline & Superior e inferior finos & 11 & 36,7 \\
\hline \multirow[t]{2}{*}{ Postura dos lábios } & Com vedamento & 27 & 90,0 \\
\hline & Sem vedamento & 3 & 10,0 \\
\hline \multirow[t]{2}{*}{ Tonicidade labial } & Normal & 12 & 40,0 \\
\hline & Hipofuncionantes & 18 & 60,0 \\
\hline \multirow[t]{2}{*}{ Mobilidade labial } & Normal & 13 & 43,3 \\
\hline & Alterado & 17 & 56,7 \\
\hline \multirow[t]{2}{*}{ Tremor de lábios } & Presente & 6 & 20,0 \\
\hline & Ausente & 24 & 80,0 \\
\hline \multicolumn{4}{|l|}{ Língua } \\
\hline \multirow[t]{2}{*}{ Morfologia da língua } & Normal & 18 & 60,0 \\
\hline & Alargada & 12 & 40,0 \\
\hline \multirow[t]{2}{*}{ Marcas nas laterais da língua } & Presente & 11 & 36,7 \\
\hline & Ausente & 19 & 63,3 \\
\hline \multirow[t]{2}{*}{ Frênulo lingual } & Normal & 28 & 93,3 \\
\hline & Curto & 2 & 6,7 \\
\hline \multirow[t]{3}{*}{ Postura lingual } & Região da papila & 20 & 66,7 \\
\hline & Região do assoalho bucal & 9 & 30,0 \\
\hline & Entre os dentes & 1 & 3,3 \\
\hline \multirow[t]{2}{*}{ Tonicidade lingual } & Normal & 10 & 33,3 \\
\hline & Hipotensa & 20 & 66,7 \\
\hline \multirow[t]{2}{*}{ Mobilidade lingual } & Normal & 12 & 40,0 \\
\hline & Alterada & 18 & 60,0 \\
\hline \multirow[t]{2}{*}{ Tremor de língua } & Presente & 7 & 23,3 \\
\hline & Ausente & 23 & 76,7 \\
\hline
\end{tabular}




\begin{tabular}{llcc}
\hline \multicolumn{1}{c}{ Estruturas } & \multicolumn{1}{c}{ Avaliação } & $\mathrm{n}$ & $\%$ \\
\hline Bochechas & & 21 & 70,0 \\
Simetria das bochechas & Simétricas & 9 & 30,0 \\
& Assimétricas & & \\
Marcas ou ferimentos internos nas & & 3 & 10,0 \\
bochechas & Presente & 27 & 90,0 \\
& Ausente & 3 & 10,0 \\
Morfologia das bochechas & Direita mais caída & 6 & 20,0 \\
& Esquerda mais caída & 21 & 70,0 \\
& Ambas caídas & 7 & 23,3 \\
Tonicidade das bochechas & Normal & 23 & 76,7 \\
& Hipofuncionantes & 13 & 43,3 \\
Mobilidade das bochechas & Normal & 17 & 56,7 \\
\hline
\end{tabular}

Referente ao músculo mentual, 15 idosos $(50 \%)$ apresentaram hiperfunção dessa estrutura. Quanto à morfologia do palato duro, o mesmo encontra-se normal em 28 participantes (93,3\%) e alterado em apenas dois $(6,7 \%)$. O palato mole apresentou-se morfologicamente normal em todos os componentes da amostra e verificouse bom funcionamento velofaríngeo em 29 voluntários $(96,7 \%)$ e em apenas um $(3,3 \%)$ a função mostrou-se alterada.

Quanto aos aspectos da articulação temporomandibular (ATM), referentes à mobilidade de mandíbula, verificou-se que, dos idosos avaliados, cinco $(16,7 \%)$ possuíam mobilidade normal, enquanto $25 \quad(83,3 \%)$ apresentavam desvio na abertura e/ou no fechamento. Nenhum idoso possuía crepitações mandibulares; 22 (73,3\%) possuíam estalidos; e 28 sujeitos $(93,3 \%)$ negaram a presença de dor na mandíbula.

Referente à situação dentária, metade da população estudada $(50 \%)$ apresentava edentulismo. Entre o restante da amostra, a média de dentes na arcada superior era de 1,7 com desvio-padrão de 3,1; já na arcada inferior a média era de 3,5 dentes, com desvio-padrão de 4,2. Observou-se regular estado de conservação dentária em 16 idosos (53,3\%) e, estado ruim, em 14 (46,7\%). Nenhum idoso apresentou bom estado de conservação. Quanto ao hábito de higiene oral e da prótese, 15 entrevistados $(50 \%)$ referiram realizar sempre e os outros $15(50 \%)$ afirmaram realizar às vezes. Em relação à adaptação das próteses dentárias, $16(53,3 \%)$ possuíam próteses totais bem adaptadas, enquanto 17 (56,7\%) apresentaram má adaptação da mesma (resposta múltipla). As próteses parciais estavam bem adaptadas em nove $(30 \%)$ dos componentes da amostra e mal adaptadas em quatro (13,3\%). Verificouse que cinco indivíduos (16,7\%) não utilizavam próteses.

A tabela 4 apresenta o desempenho das funções avaliadas e as alterações encontradas. 
Tabela 4. Desempenho e alterações das funções de mastigação e deglutição. Canoas-RS, 2012.

\begin{tabular}{|c|c|c|c|}
\hline Função & Avaliação & $\mathrm{n}$ & $\%$ \\
\hline \multirow[t]{6}{*}{ Mastigação } & Bilateral alternada & 1 & 3,3 \\
\hline & $\begin{array}{l}\text { Bilateral alternada com } \\
\text { predomínio lado direito }\end{array}$ & 7 & 23,3 \\
\hline & $\begin{array}{l}\text { Bilateral alternada com } \\
\text { predomínio lado esquerdo }\end{array}$ & 2 & 6,7 \\
\hline & Bilateral simultânea & 7 & 23,3 \\
\hline & Unilateral direita & 7 & 23,3 \\
\hline & Unilateral esquerda & 6 & 20,0 \\
\hline \multirow[t]{3}{*}{ Ritmo mastigatório } & Normal & 7 & 23,3 \\
\hline & Lento & 17 & 56,7 \\
\hline & Rápido & 6 & 20,0 \\
\hline \multirow[t]{2}{*}{ Lábios durante a mastigação } & Fechados & 22 & 73,3 \\
\hline & Abertos & 8 & 26,7 \\
\hline \multirow[t]{2}{*}{$\begin{array}{l}\text { Participação exagerada da musculatura } \\
\text { perioral na mastigação }\end{array}$} & Presente & 21 & 70,0 \\
\hline & Ausente & 9 & 30,0 \\
\hline \multirow[t]{2}{*}{ Movimentos de mandíbula na mastigação } & Movimentos rotatórios & 23 & 76,7 \\
\hline & Movimentos verticais & 7 & 23,3 \\
\hline \multirow[t]{2}{*}{ Deglutição } & Normal & 12 & 40,0 \\
\hline & Adaptada & 18 & 60,0 \\
\hline \multirow[t]{2}{*}{ Projeção anterior de língua } & Presente & 6 & 20,0 \\
\hline & Ausente & 24 & 80,0 \\
\hline \multirow[t]{2}{*}{$\begin{array}{l}\text { Participação exagerada da musculatura } \\
\text { perioral na deglutição }\end{array}$} & Presente & 18 & 60,0 \\
\hline & Ausente & 12 & 40,0 \\
\hline
\end{tabular}

$\mathrm{Na}$ avaliação da deglutição, não foram observados sinais de suspeita de disfagia como engasgo e tosse, na população estudada.
Na tabela 5 estão apresentados os resultados (teste Exato de Fisher) que verificam associação estatisticamente significativa da ocorrência de desvio mandibular com os indivíduos que não possuíam a prótese dentária total superior bem adaptada e com a presença de estalido. 
Tabela 5. Dados da relação entre desvio mandibular e prótese dentária total e estalido. Canoas-RS, 2012.

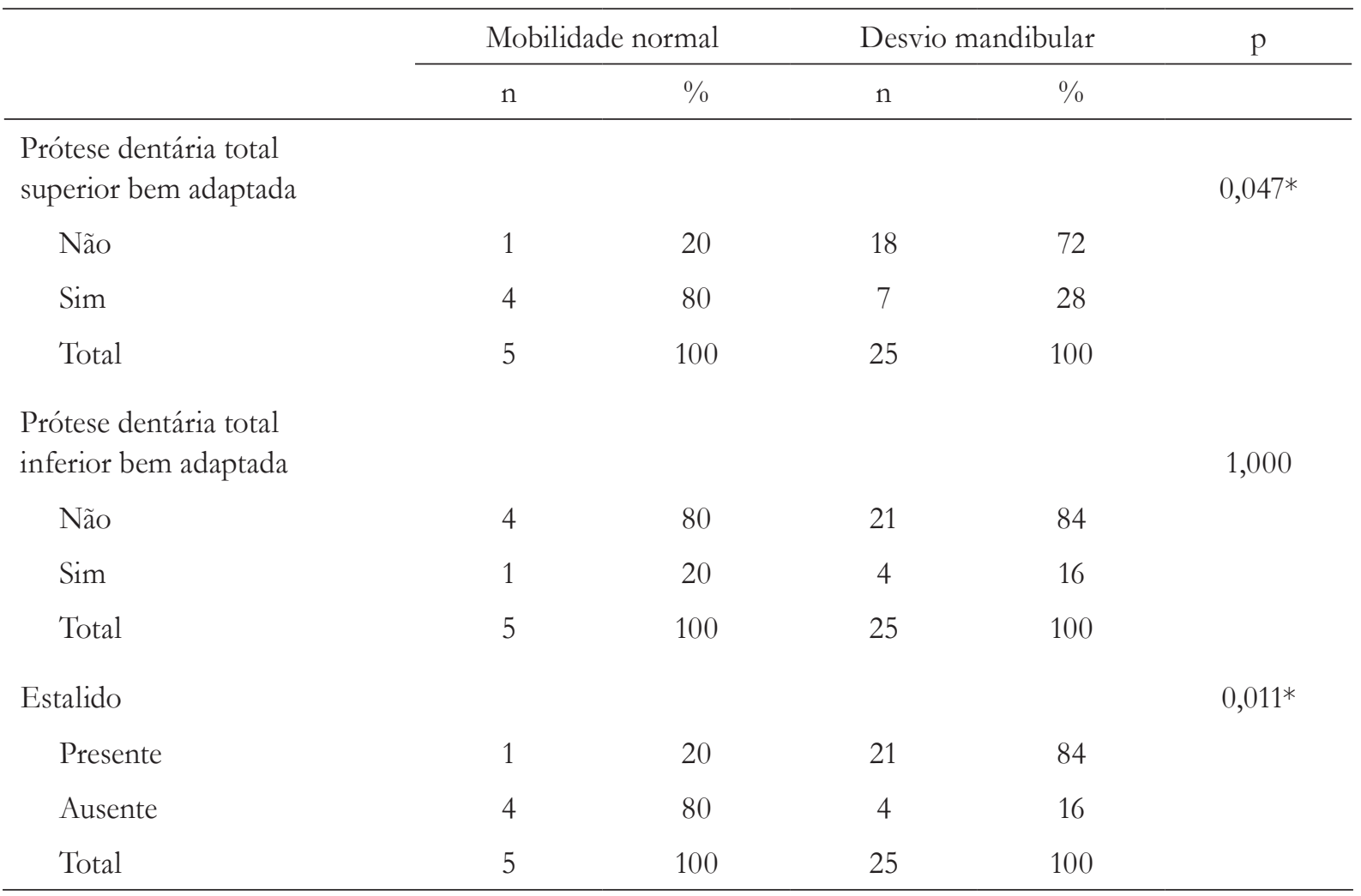

*Teste exato de Fisher $\mathrm{p}<0,05$ (significante).

\section{DISCUSSÃO}

Com o passar dos anos, as estruturas do sistema estomatognático e suas funções acabam sofrendo modificações, as quais fazem parte do processo natural de envelhecimento, levando o idoso a adaptar-se diante de suas condições morfofuncionais. Frente aisso, torna-se necessário conhecer o perfil e as demandas dessa população, com o intuito de auxiliar no planejamento das ações fonoaudiólogicas. ${ }^{5,15}$ Nessa amostra, houve predomínio do gênero feminino e baixo nível de escolaridade, corroborando a literatura nacional e refletindo os dados brasileiros quanto ao perfil dos idosos institucionalizados..$^{16-18}$

Durante a entrevista realizada com os participantes, no que se refere a saúde atual e fatores associados, verificou-se que a hipertensão arterial está entre as doenças mais citadas, dado que corrobora a literatura consultada. ${ }^{13,18}$ Quanto ao consumo de álcool e cigarro, observou-se que grande número de indivíduos nunca fez uso dos mesmos. São poucos os estudos que investigam a prevalência do uso dessas substâncias em idosos residentes de instituições de longa permanência para idosos (ILPIs). Estudo realizado por Lima et al. ${ }^{13}$ verificou baixa prevalência do consumo de álcool entre os sujeitos pesquisados, mas a utilização de cigarros foi considerada alta pelos autores, sendo que 38\% da amostra eram fumantes.

A dificuldade em mastigar algum tipo de alimento mostrou-se bastante frequente, uma vez que $56,7 \%$ da população estudada referem esta 
queixa sempre e 16,7\% às vezes. Essa dificuldade pode ser explicada pela diminuição do tônus muscular e coordenação da musculatura envolvida na mastigação, pela perda da dentição natural e pela má adaptação de próteses dentárias, sendo que estes fatores afetam diretamente a preparação do bolo alimentar e ocasionam maior dificuldade com alimentos duros e fibrosos. ${ }^{9,17}$ Em pesquisa realizada por Dias \& Cardoso,${ }^{15}$ a fim de analisar as características miofuncionais orofaciais relacionadas à deglutição em um grupo de 15 idosas institucionalizadas, $80 \%$ relatam apresentar dificuldade para com alimentos sólidos.

Justificada pelo déficit mastigatório, a preferência por alimentos macios foi outro aspecto referido pela amostra, caracterizando uma estratégia de adaptação frente às mudanças morfológicas efuncionais. ${ }^{7,12,19}$ Em estudorealizado por Jales et al., ${ }^{7}$ que identificou as características do sistema estomatognático apontadas por idosos de instituição pública e privada, constatou-se que a maioria $(85 \%$ dos idosos da instituição pública e $65 \%$ da privada) afirmou preferir os alimentos mais macios ou pastosos, achado que coincide com os da presente pesquisa.

Mais da metade dos indivíduos entrevistados afirmou possuir a necessidade de temperar mais a comida para conseguir perceber o sabor. Este fato foi citado em pesquisa realizada por Lima et al., ${ }^{13}$ que demostrou que a queixa é bastante frequente entre os idosos. A literatura ${ }^{8,9,13}$ relaciona esta necessidade à diminuição do número de botões gustativos nas papilas linguais e à higiene oral prejudicada, dificultando a estimulação dos quimiorreceptores orais.

Estudos têm apontado a insatisfação dos idosos institucionalizados, com relação ao sabor das refeições ofertadas, e constatado que a apreciação da alimentação está relacionada a atributos presentes no alimento, como sabor e tempero. Frente a isso, os autores alertam ainda quanto à monotonia no planejamento dos cardápios nas ILPIs, salientando que as refeições oferecidas devem contemplar as preferências alimentares dos idosos, a fim de que não ocorra o decréscimo do apetite, proporcionando um estado nutricional equilibrado e melhor qualidade de vida. ${ }^{20,21}$
$\mathrm{Na}$ amostra estudada, constatou-se que as alterações de mobilidade e tonicidade da musculatura de lábios, língua e bochechas são frequentes. Diversas pesquisas ${ }^{13-15}$ têm apontado resultados semelhantes aos do presente estudo. Rocha \& Lima ${ }^{17}$ confirmam os dados em sua pesquisa, segundo a qual $77 \%$ dos idosos avaliados verificaram a ocorrência de déficit na mobilidade das bochechas e $85 \%$ apresentaram tônus alterado das mesmas. Quanto à língua, foi observado que $62 \%$ apresentaram alteração de tonicidade e $85 \%$ demonstraram alguma dificuldade de mobilidade. Referente aos lábios, as autoras encontraram percentagem de $62 \%$ para alteração de tônus e $69 \%$ para déficit de mobilidade desta estrutura. De acordo com a literatura, essas alterações ocorrem devido à diminuição de massa, do número de unidades motoras e de força muscular, bem como do controle dos movimentos, situação característica do processo de envelhecimento das estruturas orofaciais. ${ }^{9,13,15}$

Quanto ao aspecto dentário, todos os participantes deste estudo apresentaram um número de dentes naturais bastante reduzido, sendo que, destes, $50 \%$ são edêntulos totais e mais da metade possui próteses dentárias mal adaptadas. $O$ fato de nenhum sujeito ter apresentado estado de conservação adequado é outro dado relevante. Estes achados estão de acordo com diversos estudos ${ }^{8,14,18}$ realizados em populações idosas brasileiras, os quais demonstram elevada prevalência de problemas bucais, como cáries, perdas dentárias, edentulismo, dispensa do uso ou dificuldades na adaptação de próteses e higiene bucal precária, evidenciando a falta de programas de promoção e prevenção voltados a esta população.

Sabe-se que a condição dentária influencia no processo de alimentação, interferindo na função de mastigação, afetando o preparo do bolo alimentar e, consequentemente, dificultando sua deglutição, situação que muitas vezes ocasiona no idoso desconforto, diminuição da ingesta de alimentos sólidos e perda do prazer em se alimentar. ${ }^{10,12,17}$

No que diz respeito à mobilidade de mandíbula dos indivíduos avaliados, verificou- 
se elevada prevalência de sinais sugestivos de disfunção temporomandibular (DTM), sendo que $73,3 \%$ apresentaram estalidos e em 83,3\% foram observados desvios mandibulares. Os dados corroboram estudo anterior realizado por Canterji et al., ${ }^{22}$ o qual observou prevalência de 61\% da presença de ruídos articulares em pacientes idosos institucionalizados e Almeida et al., ${ }^{23}$ que constataram prevalência de 60\% de idosos portadores de DTM. Por outro lado, os resultados obtidos diferem dos achados de Carvalho et al., ${ }^{24}$ os quais indicam que apenas $20 \%$ dos idosos que compunham a amostra apresentaram sinais sugestivos de alterações na articulação temporomandibular (ATM). Cabe ressaltar que os estudos de Almeida et al. ${ }^{23} \mathrm{e}$ Carvalho et al. $^{24}$ foram realizados com idosos não institucionalizados.

$\mathrm{Na}$ presente pesquisa, foi verificada associação estatisticamente significativa da ocorrência de desvio mandibular com os idosos que não possuem a prótese total superior bem adaptada e com a presença de estalido. Bontempo \& Zavanelli ${ }^{25}$ realizaram estudo a fim de comparar a prevalência de sinais e sintomas de DTM em relação a seus fatores etiológicos em pacientes portadores de próteses totais bimaxilares, com idades entre 44 e 90 anos. Apesar de os indivíduos pesquisados não terem sido subdivididos em grupos etários, os autores concluíram que os sujeitos que usavam próteses totais em condições clínicas adequadas apresentaram menos sinais e sintomas de DTM do que os que usavam próteses totais em condições clínicas insatisfatórias. Já em outro estudo, realizado por Shibayama et al., ${ }^{26}$ o qual também não subdivide a amostra em grupos etários, os autores encontraram maior prevalência de disfunção temporomandibular no grupo de pacientes dentados (51\%) do que no dos pacientes usuários de próteses totais duplas.

É citado na literatura que a falta de dentes posteriores e a utilização de próteses removíveis mal adaptadas são fatores etiológicos para a disfunção temporomandibular. A redução da dimensão vertical de oclusão aumenta a sobrecarga mecânica na ATM e o uso de próteses com deficiência de estabilidade e retenção favorecem o aparecimento de hábitos parafuncionais como o apertamento dentário. Sendo assim, a articulação do idoso que já possui uma capacidade de regeneração reduzida tende a sofrer desgaste de seus componentes articulares. ${ }^{27}$

Alguns estudos referem que, apesar de os sinais de DTM aumentarem nos idosos, as queixas de disfunções e sintomas tendem a diminuir com o avançar da idade. Esta condição pode ser explicada com a concepção de que os idosos aceitam esses sinais como próprios do envelhecimento e não os encaram como patologia, adaptando-se às funções orais diminuídas e associando o desconforto as suas próteses dentárias. ${ }^{22,26}$ Logo, observam-se ainda muitas discrepâncias na literatura científica quanto à prevalência de sinais de DTM em idosos, devido às diferentes metodologias empregadas. Sendo assim, sugere-se a realização de estudos mais específicos abordando o tema.

No que diz respeito à avaliação das funções estomatognáticas, verificou-se, na maioria dos idosos pesquisados, a participação exagerada da musculatura perioral, tanto na mastigação, quanto na deglutição. $\mathrm{O}$ aumento no uso desta musculatura caracteriza uma adaptação na tentativa de manipular melhor o bolo alimentar dentro da cavidade oral, uma vez que o músculo mentual entra em hiperfunção, compensando a musculatura periorbicular hipofuncionante. 9,10

O ritmo mastigatório lentificado mostrou-se frequente entre os participantes deste estudo, corroborando a literatura consultada, que afirma que com o envelhecimento, as ações motoras tendem a se tornar mais lentas e descoordenadas, podendo este fato estar relacionado, ainda, aos aspectos dentários do idoso. ${ }^{10,12}$

Embora aproximadamente um terço da amostra tenha autorreferido a presença de engasgo e tosse às vezes, a não verificação, na avaliação, de sinais de suspeita de disfagia pode ser justificada pelo fato de ter sido realizada apenas uma observação da refeição de cada idoso, limitando assim, a ocorrência e, pelos critérios de elegibilidade utilizados, os quais eliminaram indivíduos com déficits e doenças de base que 
muitas vezes resultam numa deglutição alterada, configurando-se como limitações deste estudo.

\section{CONCLUSÃO}

Com base nos resultados do estudo, podese concluir que o perfil da amostra pesquisada condiz com o da população institucionalizada nacionalmente: as principais modificações observadas nas estruturas estomatognáticas e em suas funções foram as alterações de mobilidade e tonicidade e a adaptação da mastigação e da deglutição, por meio da participação exagerada da musculatura perioral e do ritmo mastigatório lentificado; as dificuldades alimentares mais relevantes, autorreferidas pelos idosos, dizem respeito à restrição de aceitação de alimentos

\section{REFERÊNCIAS}

1. Silva AECS, Menezes EAG, Coelho TOA, Moraes EN. Aspectos Bio-Psico-Sociais dos Idosos Institucionalizados na Casa do Ancião da Cidade Ozanan, no Ano de 2005, em Belo Horizonte. In: Anais do $8^{\circ}$ encontro de extensão da UFMG. Belo Horizonte - 03 a 08 de outubro de 2005. [online] Disponível em: http://www.ufmg.br/proex/arquivos/8Encontro/ Saude_7.pdf. Data de acesso: 23/03/2012.

2. Born T, Boechat N. A qualidade dos cuidados ao idoso institucionalizado. In: Freitas E, et al. Tratado de Geriatria e Gerontologia. $1^{a}$ ed. Rio de Janeiro: Guanabara Koogan, 2002.

3. Camarano AA, Kanso S. As instituições de longa permanência para idosos no Brasil. Revista Brasileira de Estudos de População. 2010;1(27):233-235 [online] Disponível em: http://www.abep.nepo. unicamp.br/docs/rev_inf/vol27_n1_2010/vol27_ n1_2010_notapesquisa_p233a235.pdf. Data de acesso: 23/03/2012.

4. Ribeiro A. Aspectos biológicos do envelhecimento. In: Russo IP. Intervenção Fonoaudiológica na Terceira Idade. Rio de Janeiro: Revinter, 2004.

5. Feijó AV, Rieder, CRM. Distúrbios da deglutição em idosos. In: Jacobi JS, Levy DS, Silva LMC. Disfagia: Avaliação e tratamento. $3^{\text {a }}$ ed. Rio de Janeiro: Revinter, 2004.

6. Douglas CR. Patologia oral: fisiologia normal e patológica aplicada à odontologia e fonoaudiologia. São Paulo: Pancast; 1998 mais sólidos, já que a dificuldade em mastigar algum alimento e a preferência por alimentos macios foram citadas mais frequentemente. $\mathrm{O}$ engasgo e a tosse foram referidos como presentes, às vezes, por um terço da amostra, porém não foram observados na avaliação.

Os resultados da pesquisa evidenciam a necessidade da atuação fonoaudiológica interdisciplinar nas instituições de longa permanência para idosos, a fim de minimizar o impacto dos efeitos do envelhecimento nas funções estomatognáticas, proporcionando aos idosos melhor qualidade de vida.

Sugere-se a realização de novos estudos que busquem estratégias para o desenvolvimento de ações fonoaudiológicas efetivas nessa população.
7. Jales MA, Cabral RR, Silva HJ, Cunha DA. Características do sistema estomatognático em idosos: diferenças entre instituição pública e privada. Rev CEFAC, 2005;7(2):178-87.

8. Catão MHCV, Xavier AFC, Pinto TCA. O impacto das alterações do sistema estomatognático na nutrição do idoso. Rev Bras Ciênc Saúde. 2011;9(29):73-8.

9. Amaral AKFJ. Interface da motricidade orofacial com a gerontologia. In: Pernambuco LA. Atualidades em Motricidade Orofacial / Org. Pernambuco LA et. al; Rio de Janeiro: REVINTER, 2012.

10. Marchesan IQ. Distúrbios da motricidade oral. In: Russo IP. Intervenção fonoaudiológica na terceira idade. Rio de Janeiro: Revinter, 2004.

11. Amaral AKFJ, Silva HJ, Cabral ED. Fatores determinantes do tempo de maceração dos alimentos em idosas edêntulas totais. Rev CEFAC, 2009;11 Supl3:S398-404. [online] Disponível em: http://www.scielo.br/scielo.php?script=sci_arttext \&pid=S1516-18462009000700016. Data de acesso: 07/04/2012.

12. Cardos MCAF, Bujes RV. A Saúde bucal e as funções de mastigação e deglutição nos idosos. Estudos Interdisciplinares sobre Envelhecimento. Porto Alegre 2010;1(15).

13. Lima RMF, Amaral AKFJ, Aroucha EBL, Vasconcelos TMJ, Silva HJ, Cunha DA. Adaptações na mastigação, deglutição e fonoarticulação em idosos de instituição de longa permanência. Rev CEFAC, 
2009;11(supl3):405-22. [online] Disponível em: http:// www.scielo.br/pdf/rcefac/v11s3/a17v11s3.pdf. Data de acesso: 23/03/2012.

14. Marcolino J, Czechowski AE, Venson C, Bougo GC, Antunes KC, Tassinari N, et al. Achados fonoaudiológicos na deglutição de idosos do município de Irati - Paraná. Rev Bras Geriatr Gerontol. 2009;12(2):193-200. [online] Disponível em: http://www.crde-unati.uerj.br/img_tse/v12n2/pdf/ art_3.pdf. Data de acesso: 07/04/2012.

15. Dias BKP, Cardoso MCAF. Características da função de deglutição em um grupo de idosas institucionalizadas. Estudos Interdisciplinares sobre o Envelhecimento, Porto Alegre, 2009;1(14).

16. Davim RMB, Torres GV, Dantas SMM, Lima VM. Estudo com idosos de instituições asilares no município de Natal/RN: características socioeconômicas e de saúde. Rev Latino-am Enfermagem, mai/jun 2004;12(3):518-24.

17. Rocha MAS, Lima MLLT. Caracterização dos distúrbios miofuncionais orofaciais de idosos institucionalizados. Rev Bras Geriatr Gerontol. 2010; 4 (1):21-6.

18. Roque FP, Bomfim FMS, Chiari BM. Descrição da dinâmica de alimentação de idosas institucionalizadas. Revista da Sociedade Brasileira de Fonoaudiologia. 2010;15(2):256-63.

19. Suzuki HS. Modificações nos hábitos da rotina alimentar do indivíduo idoso normal. Rev Soc Bras Fonoaudiol 1998; 2(3):43-7.

20. Sass A, Marin CF, Hattanda C, Semprebom KA, Zibordi RQ, Kaneshima EN, Kaneshima AMS. Qualidade de vida e padrão alimentar de idosos institucionalizados na cidade de Maringá-PR. Iniciação Científica CESUMAR jul.dez. 2004;2(6):120-125.
21. Santelle O, Lefrève AMC, Cervato AM. Alimentação institucionalizada e suas representações sociais entre moradores de instituições de longa permanência para idosos em São Paulo. Cad. Saúde Pública, Rio de Janeiro, dez 2007;23(12):3061-3065.

22. Canterji MB, Amenábar JM, Lima LK, Padilha DMP, Sousa ACA. Frequência de sinais clínicos e sintomas de disfunções temporomandibulares em pacientes idosos institucionalizados. R. Fac. Odonto., Porto Alegre, jul. 2004;1(45):48-51.

23. Almeida LHM, Farias ABL, Soares MSM, Cruz JSA, Cruz RES, Lima MG. Disfunção temporomandibular em idosos. RFO, jan/abr 2008;1(13):35-38.

24. Carvalho KC, Lélis EM, Carvalho NF, Ferreira DLA, Rocha GM, Souza APS, et al. Prevalência dos sinais e sintomas sugestivos de disfunções temporomandibulares em um grupo de idosos da cidade de Teresina. ConScientiae Saúde, 2010;9(3):441-447.

25. Bontempo KV, Zavanelli RA. Fatores etiológicos correlacionados à desordem temporomandibular em pacientes portadores de próteses totais bimaxilares: uma análise comparativa. RFO, Porto Alegre, jan/ mar 2009;1(57):67-75.

26. Shibayama R, Contreiras E, Shibayama B, Sella M, Boer PR, Nakui MT. Prevalência de disfunção temporomandibular em pacientes portadores de próteses totais duplas. Revista Odontológica de Araçatuba, jul/dez 2008;2(29):46-51.

27. Santos-Daroz CB, Senna PM, Nuñez JMC, Lucena SC, Barbosa CMR. Relação entre o envelhecimento, problemas articulares e disfunção temporomandibular. Revista Brasileira de Pesquisa em Saúde, 2009;11(1):46-51. 